\title{
European Society for Medical Oncology 2020
}

\author{
Rupert Bartsch
}

The 2020 Congress of the European Society for Medical Oncology (ESMO) was another large meeting held solely in a virtual format due to the ongoing severe acute respiratory syndrome coronavirus 2 (SARS-CoV2) pandemic. While we all have-to a certain extent-become used to the transfer of large parts of our professional lives into the virtual world, following developments outside our own field of expertise has become more difficult. Therefore, reviews focusing on congress highlights have gained importance and in the current issue, the Magazine of European Medical Oncology (MEMO) provides a series of articles focusing on relevant results presented at the virtual 2020 ESMO congress in the fields of breast cancer, nonsmall-cell lung cancer (NSCLC), gastrointestinal and genitourinary cancers.

Drs. Popper and Rumpold present data on esophageal and gastric cancer and not surprisingly, the focus lies with immunotherapy [1]. Data from CheckMate 577 were presented at a Presidential Symposium: In patients with resected esophageal or gastro-esophageal junction cancers after neoadjuvant chemoradiotherapy, treatment with the immune checkpoint inhibitor nivolumab yielded a statistically significant and clinically relevant prolongation of disease-free survival (DFS) over placebo. While data on overall survival (OS) are still awaited, these data may eventually become practice changing.

Data on pancreatic cancer are summarized by Dr. Bergen, and promising results of several novel targeted approaches were presented [2]. On the downside, a randomized phase II trial found no benefit for the addition of combination immunotherapy con-

\section{Dr. R. Bartsch $(\bowtie)$}

Department of Medicine I, Medical University of Vienna, Währinger Gürtel 18-20, 090 Vienna, Austria rupert.bartsch@meduniwien.ac.at sisting of durvalumab and tremelimumab to first-line chemotherapy, again indicating the relative treatment resistance of pancreatic cancer.

Treatment of NSCLC was revolutionized in recent years and Dr. Kiesewetter summarizes the most recent developments [3]. At the 2020 ESMO congress, the focus was not on immunotherapy but on tyrosine kinase inhibitors (TKIs) with the ADAURA trial being a shining example: Adjuvant therapy with osimertinib was already shown to improve DFS over placebo in resected NSCLC harbouring activating EGFR mutations. Central nervous system (CNS) DFS was a predefined secondary endpoint of this study, and the risk for CNS relapses was reduced by an impressive $82 \%$.

Finally, in breast cancer, early results of the monarchE trial suggested a benefit of adding the cyclindependent kinase (CDK) 4/6 inhibitor abemaciclib to standard adjuvant endocrine therapy in high-risk hormone-receptor positive early stage breast cancer [4]. While these data are intriguing, the PALLAS trial could not establish a comparable benefit with the CDKi palbociclib in a similar setting making final conclusions impossible at this stage. Results from the ASCENT trial in pretreated patients with metastatic triple-negative breast cancer are immediately practice changing once the antibody-drug conjugate (ADC) sacituzumab-govitecan is available in Europe. In this phase III trial, the ADC yielded a clinically relevant improvement of DFS and OS over chemotherapy by physician's choice.

In summary, data from several highly relevant studies were presented at the 2020 ESMO congress, which shows the importance that this meeting has meanwhile achieved.

Conflict of interest R. Bartsch: Advisory role: Astra-Zeneca, Daiichi, Eisai, Eli-Lilly, MSD, Novartis, Pfizer, Pierre-Fabre, Puma, Roche; lecture honoraria: Astra-Zeneca, Eli-Lilly, No- 
vartis, Pfizer, Pierre-Fabre, Roche; research support: Daiichi, MSD, Novartis, Roche.

\section{References}

1. PopperU,RumpldH.UpdateESMO: gastricandesophageal cancer. memo. 2021;14(2). https://doi.org/10.1007/ s12254-021-00694-5.

2. Bergen ES. ESMO 2020 update: Pancreatic cancer. memo. 2021;14(2). https://doi.org/10.1007/s12254-021-00692-7.

3. Kiesewetter B. ESMO virtual congress 2020-highlights NSCLC. memo. 2021;14(2). https://doi.org/10.1007/ s12254-021-00700-w.

4. Bartsch R. ESMO 2020: highlights in breast cancer. memo. 2021;14(2). https://doi.org/10.1007/s12254-021-00703-7.
Publisher's Note Springer Nature remains neutral with regard to jurisdictional claims in published maps and institutional affiliations.

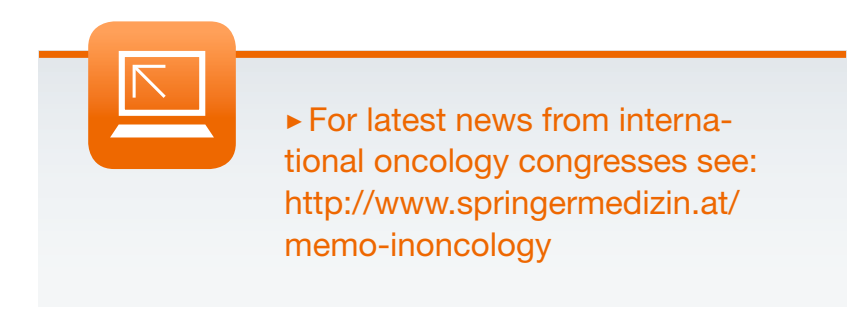

